\title{
Desarrollo de un cemento de base silicatada a partir de rocas volcánicas vítreas alcalinas: interpretación de los resultados preindustriales basada en la composición químico-mineralógica de los precursores geológicos
}

\author{
D.GIMENO ${ }^{(1)}$, J.DAVIDOVITS ${ }^{(2)}$, C.MARINI ${ }^{(3)}$, P.ROCHER ${ }^{(4)}$, S.TOCCO ${ }^{(4)}$, S.CARA ${ }^{(4)}$, N.DIAZ ${ }^{(1)}$, C.SEGURA ${ }^{(1)}$, G.SISTU ${ }^{(6)}$ \\ (1) Dept. Geoquímica, Petrologia i Prospecció Geològica, Fac. Geologia, Universitat de Barcelona, 08028 - Barcelona. Domingo@ natura.geo.ub.es \\ (2) Geopolymer Institut \& Cordi-Géopolymère, Saint Quentin, Francia \\ ${ }^{{ }^{3}}$ Università di Cagliari, Dept. Scienze della Terra, Cagliari, Italia \\ (4) B.R.G.M. Bureau de Recherches Géologiques et Minières, BRGM Auvergne, Aubiere, Francia \\ ${ }^{(5)}$ DIGITA, Università di Cagliari, Cagliari, Italia \\ (6) Dipto. Ricerche Economiche e Sociali, Università di Cagliari, Cagliari, Italia
}

\begin{abstract}
El proyecto GEOCISTEM intentó hallar un substituto vítreo alcalino natural, económico y viable industrialmente, a los reactivos químicos empleados en un cemento silicatado patentado. Se realizó una completa prospección de los recursos consistentes en rocas volcánicas vítreas ricas en álcalis $\left(\mathrm{Na}_{2} \mathrm{O}+\mathrm{K}_{2} \mathrm{O}>10 \%, \mathrm{~K}>>\mathrm{Na}\right)$, preferentemente fragmentarias (piroclásticas) en diferentes regiones volcánicas europeas (Italia, Grecia, España). Unas 100 muestras fueron analizadas (elementos mayores mediante FRX; caracterización, petrográfica y mediante DRX) y 10 fueron empleadas en la fabricación (en laboratorio y escala semiindustrial) del cemento silicatado. Se obtuvo toda una familia de cementos (diez) con alta resistencia a la compresión (50-60 MPa a los 28 días), resistentes a la corrosión y que no desarrollan reacción alcalina-agregados, muy adecuados para el encapsulado de residuos especiales; todo ello con una notable reducción del consumo de energía en el proceso de fabricación y en el consumo de silicato de K (hasta 1/3-1/4 del requerido en la patente original). El estudio químico-mineralógico desarrollado demuestra que la formulación original del cemento era excesivamente restrictiva, y que la mineralogía producida en los procesos de desvitrificación naturales controla estrictamente el rendimiento de estos nuevos recursos durante el proceso de fabricación del cemento. Las rocas anhidras con feldespatos alcalinos y fases silíceas cristalinas predominantes obtenidas a temperaturas inferiores a las magmáticas (desvitrificación) son más interesantes que las zeolitizadas naturalmente, ya que no requieren calcinación previa con el consiguiente ahorro energético.
\end{abstract}

Palabras clave: cemento silicatado, rocas volcánicas alcalinas, vidrio natural, composición química, control mineralógico

Development of silicate-based cement from glassy alkaline volcanic rocks: interpretation of preliminary data related to chemical-mineralogical composition of geologic raw materials

The GEOCISTEM project was focussed to look for an inexpensive natural glassy alkaline substitute of chemical reagents used in a trade registered cement. A complete survey for european resources made up for fragmentary (pyroclastic) alkali-rich glassy volcanic rocks $\left(\mathrm{Na}_{2} \mathrm{O}+\mathrm{K}_{2} \mathrm{O}>10 \%, \mathrm{~K}>>\mathrm{Na}\right)$ was done in several european volcanic regions (in Italy, Greece and Spain). Up to 100 samples were analysed (main constituents by XRF; petrographic and XRD characterisation) and 10 of them were used in the developing of a silicate-based cement (laboratory and semi-industrial scale). The result was a set (ten) of cements characterised by high compressive strength of the plain cement phase (50-60 MPa after 28 days), with high resistance to chemical corrosion and non alkali-silica-aggregate reaction, very indicated for toxic waste encapsulation. Furthermore a great economy in energy and a significant reduction of K-silicate consumption (up to 1/3-1/4 of the formulation in the original trade registered cement) in the process of production was achieved. The chemical-mineralogical study carried out shows that the original formulation of the cement was too restrictive, as well as that the mineralogy reached during natural devitrification processes in the rock strictly controls the performance of this new european resource during the development of the cement. The anhidrous rocks mainly constituted by alkali feldspars and silica crystalline phases (obtained by devetrification at temperatures under magmatic ones) are more interesting than the zeolitised ones, allowing to skip the calcination process and thus providing energetic economy.

Keywords: silicate-based cement, alkali-rich volcanic rocks, natural glass, chemical composition, mineralogical control

\section{INTRODUCCIÓN}

Este trabajo forma parte del desarrollo del proyecto GEOCISTEM, financiado por la Comisión Europea en el periodo 1994-1997; el nombre del proyecto es un acrónimo del título en inglés que puede traducirse por "Cementos geopoliméricos económicamente viables, destinados a la estabilización de elementos tóxicos" (1).

El proyecto financiado partía de una patente comercial desarrollada por uno de los participantes en el proyecto
(Cordi-Geopolymère, 2), según la cual a partir de reactivos químicos se obtiene un cemento de óptimas propiedades encapsuladoras, por lo que se refiere a los elementos tóxicos, y notablemente más resistente a los ácidos que los cementos estándar de base carbonatada. Además, este cemento presenta un gran potencial medioambiental, si se tiene en cuenta que, de desarrollarse su producción en términos económicos competitivos con los cementos normales de base carbonatada 
podría llegar a substituirlos en gran medida en la industria de la construcción.

Este segundo aspecto no es marginal desde el punto de vista ambiental, ya que algunos autores han contabilizado que se llega a emitir a la atmósfera cerca de una tonelada de $\mathrm{CO}_{2}$ (tanto el gas procedente de la desintegración del carbonato mineral como el procedente de la energía consumida en el proceso expresado en forma de combustión de gasóleo equivalente) durante la producción de una tonelada de cemento portland ordinario (1). Es bien conocido el efecto que este gas induce en la potenciación del llamado efecto invernadero e, igualmente, los intentos de establecimiento de una ecotasa europea desde 1991 para mitigar su producción. Sin olvidar que durante el proceso de producción del cemento con el $\mathrm{CO}_{2}$ se emite a la atmósfera particulado en suspensión y cantidades notables de metales pesados contenidos en los carbonatos naturales empleados que revierten inmediatamente sobre el ecosistema.

Podemos resumir que la patente $(1,2,3)$ se basa en la reacción química:

\section{$2\left(\mathrm{Si}_{2} \mathrm{O}_{5^{\prime}}, \mathrm{Al}_{2} \mathrm{O}_{2}\right)+\mathrm{Ca}\left(\mathrm{H}_{3} \mathrm{SiO}_{4}\right)_{2}+\mathrm{K}_{2}\left(\mathrm{H}_{3} \mathrm{SiO}_{4}\right)_{2} \longrightarrow\left(\mathrm{K}_{2} \mathrm{O}, \mathrm{CaO}\right)\left(8 \mathrm{SiO}_{2}, 2 \mathrm{Al}_{2} \mathrm{O}_{3} \mathrm{nH}_{2} \mathrm{O}\right)$}

Los reactivos originalmente empleados en la patente son un kaolín impuro calcinado, un desecho industrial (escoria de alto horno) y el silicato de potasio, el único elemento que de hecho encarece el proceso de fabricación hasta aproximadamente 0.5 euro por $\mathrm{kg}$. Desde el punto de vista práctico, el desarrollo de este proyecto esencialmente se dirigió a obtener aportes naturales alternativos de elementos alcalinos (rocas de bajo costo) en la obtención de un cemento análogo al desarrollado en el laboratorio, a su experimentación en la producción de cementos geopoliméricos (de un costo por $\mathrm{kg}$ de al menos $1 / 10$ del original), tanto en la escala del laboratorio como en la semiindustrial, teniendo especial atención en el estudio de sus propiedades técnicas, geomecánicas y químicas, al desarrollo de pellets con mezcla de absorbentes y el citado cemento que permitieran encapsular residuos tóxicos y su manipulación posterior, y en el ensayo de la durabilidad del cemento y su comparación con análogos históricos, en especial con cementos pozzolánicos de época romana.

Los aportes naturales de elementos alcalinos fueron escogidos siguiendo unos cuantos parámetros: elevado contenido en óxidos alcalinos $\left(\mathrm{Na}_{2} \mathrm{O}+\mathrm{K}_{2} \mathrm{O}>10 \%, \mathrm{~K}>>\mathrm{Na}\right)$ evitando elementos considerados penalizantes; amplia disponibilidad del tipo de roca escogido en los países de la Unión Europea, considerando tanto los socios actuales como los países en vías de serlo; facilidad de comunicación de los emplazamientos con estas rocas, y en especial proximidad a vías de comunicación acuática, atendiendo a la economía del transporte del potencial producto elaborado; abundante presencia de fase vítrea o de sus transformados naturales de un rango de temperatura formacional medio-bajo (típicamente zeolitas), de cara a economizar en los procesos de producción que requieran un elevado coste energético; y carácter clástico de ésta, de cara igualmente a economizar en procesos de trituración de la roca. Igualmente se han considerado las limitaciones ambientales y legales (áreas volcánicas consideradas parques naturales, etc.) que pudieran limitar la explotación futura de los recursos puestos en valor por el proyecto. En resumen, además de los condicionantes composicionales de las rocas las características técnico-económicas de los materiales han primado en el proceso de selección, con el resultado de que el objetivo seleccio- nado ha consistido en rocas volcánicas (elevado contenido en vidrio natural) producto de actividad eruptiva explosiva (piroclásticas) y de un rango de composición química propio de rocas altamente diferenciadas (riolitas alcalinas o peralcalinas, traquitas, fonolitas). Este trabajo se refiere principalmente al proceso de selección de las muestras, a su estudio químico-mineralógico y a la comparación de éste con los resultados de las pruebas técnicas preliminares. El cemento final desarrollado, fruto del trabajo colectivo de los participantes en el proyecto, ha sido objeto de una patente registrada a su favor por una de las empresas participantes en el proyecto (4).

\section{PROSPECCIÓN DE RECURSOS MINERALES VÍTREOS}

Del conjunto de los aspectos mencionados en el apartado anterior se concluyó en la fase preliminar del proyecto que los equipos geológicos implicados en éste (BRGM y Universidades de Cagliari y Barcelona) deberían conducir una investigación sobre los recursos de rocas volcánicas vítreas piroclásticas recientes diferenciadas (en términos amplios, cenozoicas) de Europa. Tomando como base la información cartográfica, petrológica y volcanológica se puede concluir que los principales recursos potenciales están ubicados en el área perimediterránea y en las islas Canarias. Para esta selección se consultaron las informaciones geológicas, geoquímicas y volcanológicas dispersas en varios centenares de artículos publicados en revistas especializadas, tesis doctorales, etc.; así como información inédita suministrada por las administraciones (catastros de canteras e industrias extractivas, etc.)

Dentro del área perimediterránea se consideraron principalmente los sectores del sudeste de la Península Ibérica (Cabo de Gata, sector de Mazarrón-Minas de Hellín), gran parte de las regiones volcánicas italianas (volcanismo cenozoico de la isla de Cerdeña, volcanismo plio-cuaternario de Toscana, Lazio, Campania, archipiélagos Pontino y de las Eolias) y griegas (islas de Samos, Milos, Lesbos, alrededores de Thessaloniki, volcanismo cuaternario de Macedonia hasta la frontera con la antigua República Yugoeslava) y se descartaron las regiones volcánicas francesas (principalmente por condicionantes ambientales) y alemanas (ausencia de productos geoquímicamente diferenciados, avanzado estado de agotamiento de los recursos por las industrias de los cementos hidráulicos y los abrasivos).

Otros sectores potencialmente interesantes como los archipiélagos de Azores y Madeira (Portugal) no fueron considerados por motivos económicos (lejanía de rutas comerciales internacionales, configuración de la industria cementera europea). En el archipiélago canario se consideraron inicialmente las islas de Gran Canaria, Tenerife, La Gomera y Fuerteventura, considerándose finalmente únicamente las dos primeras por motivos análogos (estructura de las rutas comerciales internacionales, condicionantes ambientales).

Tomando como base estas opciones se estructuró la prospección de los recursos geológicos en diferentes campañas de trabajo de campo, muestreo y cartografía de recursos en dos fases sucesivas desarrolladas a lo largo de los años 199495, con el resultado del muestreo y nuevos análisis químicos (elementos mayoritarios) de cerca de 100 muestras de roca. De cada muestra se tomaron rozas de un mínimo de $10 \mathrm{~kg}$, que fueron analizadas preliminarmente tras un cuarteo, disponiendo del resto de la muestra en el caso de las muestras 
seleccionadas para el desarrollo de los cementos en la escala del laboratorio. Las muestras escogidas posteriormente para los ensayos semiindustriales se remuestrearon (ya en 1996) las muestras sobre una base mínima de $100 \mathrm{~kg}$.

Aunque no es el objeto de esta nota el detallar, por prolijo, el conjunto de parámetros considerados en la selección de la información disponible, hay que señalar que se cruzaron los datos geoquímicos con los cartográficos de las unidades volcánicas, intentando en todo momento que los tipos litológicos muestreados fueran representativos de unidades lo suficientemente grandes y expuestas en la superficie (o subaflorantes) como para permitir una explotación en condiciones económicas (habida cuenta de que el precio de la materia prima debe ser especialmente bajo para favorecer el desarrollo in situ de un cemento comercializable), así como dispuestas junto a infraestructuras de transporte ya disponibles (o sobre la costa, de cara a realizar puertos sencillos de carga directamente a bodega).

Por lo que se refiere a la elección de la composición química ideal de la materia prima vítrea volcánica, las opciones posibles oscilaron en la etapa preliminar del proyecto en la búsqueda enfocada sobre composiciones lo más próximas posibles a la patente desarrollada (en la línea de los deseos de los socios industriales del consorcio de investigación) y la elección de una más amplia variedad de composiciones químicas, vítreo-mineralógicas y texturales, propugnada por los centros de investigación. Esta segunda elección presenta, además de las obvias ventajas de investigación (control del efecto de la variabilidad de diferentes parámetros de las rocas naturales en la calidad del producto final), una mayor versatilidad respecto a la potencialidad final del cemento desarrollado, y en particular del desarrollo de centros cementeros en los diferentes países comunitarios cerca de los principales centros de consumo o grandes vías comerciales. Por este motivo (ventajas en lo que se refiere a adaptabilidad del producto a condiciones locales) se pudo llegar con facilidad a un consenso respecto a la ventaja de la segunda vía y se desarrollaron (en especial en las primeras fases del proyecto) los experimentos y resultados que principalmente quedan recogidos en este trabajo.

Al final de la primera fase de prospección se seleccionaron las muestras que quedan situadas y caracterizadas geográficamente en la tabla 1 y en la figura 1.

\section{PARTE EXPERIMENTAL}

Tal como queda expresado en los apartados precedentes, la duración del estudio y la complejidad de los diferentes aspectos realizados hacen que necesariamente seamos sintéticos en los aspectos expuestos en esta nota. En concreto nos limitaremos a la exposición de las principales técnicas empleadas para la caracterización de la materia prima volcánica y el cemento geopolimérico obtenido, y quedan explícitamente excluidas ya que son objeto de un trabajo paralelo las referidas a la experimentación con arcillas esmectíticas naturales como absorbentes pelletizables.

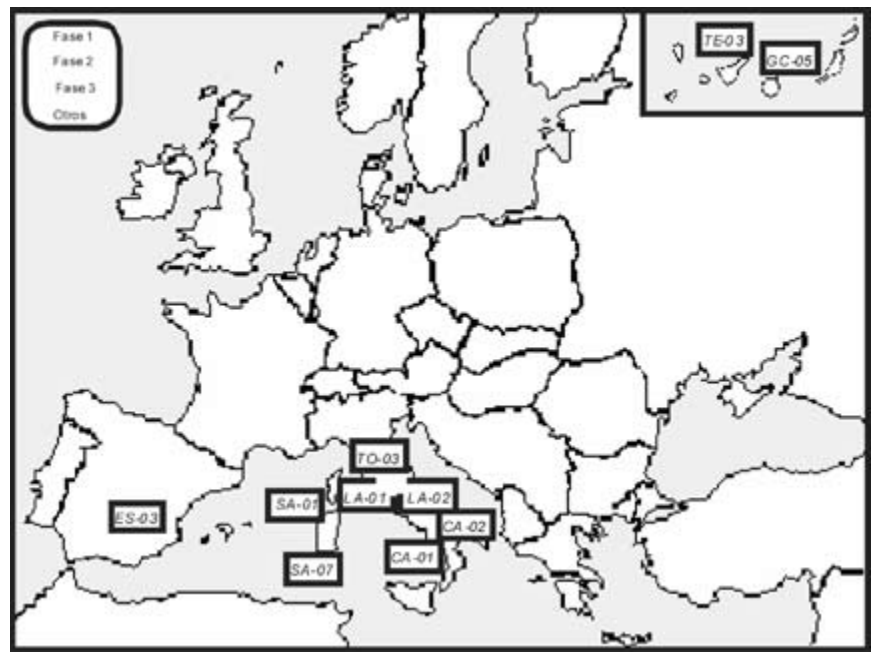

Fig. 1.- Mapa de situación de las muestras referidas en la tabla 1.

TABLA 1.- COMPOSICIÓN QUÍMICA (ELEMENTOS MAYORITARIOS, EXPRESADA EN \%) DE LAS ROCAS SELECCIONADAS PARA LA FABRICACIÓN DE CEMENTOS EN EL LABORATORIO. LOI SE REFIERE AL CONTENIDO EN VOLÁTILES MEDIDO A PARTIR DE LA PÉRDIDA AL FUEGO Y PUEDE ASIMILARSE EN ESTAS MUESTRAS A CONTENIDO EN AGUA ESTRUCTURAL. LA DESCRIPCIÓN DEL TIPO LITOLÓGICO RECOGE SUCESIVAMENTE SUS CARACTERÍSTICAS MACROSCÓPICO-PETROGRÁFICAS Y SU CLASIFICACIÓN BASADA EN EL DIAGRAMA TAS (FIG. 2) Y, EN EL CASO DE LAS TRAQUITAS, PREVIA REALIZACIÓN DE LA NORMA CIPW Y COMPROBACIÓN DE QUE EL CONTENIDO EN CUARZO NORMATIVO ES INFERIOR AL $20 \%$.

\begin{tabular}{|c|c|c|c|c|c|c|c|c|c|c|c|c|c|c|}
\hline Muestra & Localidad & Tipo petro. & $\mathrm{SiO}_{2}$ & $\mathrm{Al}_{2} \mathrm{O}_{3}$ & $\overline{\mathrm{K}_{2} \mathrm{O}}$ & $\overline{\mathrm{Na}_{2} \mathrm{O}}$ & $\mathrm{CaO}$ & $\mathrm{Fe}_{2} \mathrm{O}_{3}$ & MgO & $\overline{\mathrm{TiO}_{2}}$ & MnO & $\overline{\mathbf{P}_{2} \mathbf{O}_{5}}$ & LOI & TOTAI \\
\hline$\overline{\text { SA-01 }}$ & Milis, Montiferru, I & Lava, andesita & 71,48 & $\overline{14,55}$ & $\overline{10,41}$ & 0,69 & 0,07 & $\overline{1,6}$ & 0,04 & 0,16 & 0,01 & 0,04 & 0,96 & $\overline{99}$ \\
\hline SA-07 & Paringianu, I & Ignimbrita, riolita & 74,16 & 13,8 & 4,89 & 4,33 & 0,43 & 1,13 & 0,17 & 0,13 & 0,02 & 0,02 & 0,92 & 99 \\
\hline CA-01 & Poggiospinelli, I & Ignimbrita, traquita & 56,03 & 16,09 & 6,61 & 3,75 & 2,44 & 3,28 & 0,82 & 0,41 & 0,13 & 0,1 & 9,53 & 98 \\
\hline CA-02 & Maddaloni, I & Ignimbrit & 56,78 & $\overline{17,88}$ & 7,33 & 1,98 & 3,54 & 3,84 & 1,13 & 0,49 & 0,14 & 0,15 & 6,75 & 99 \\
\hline LA-01 & La Rocca, Tuscania, I & Ignimbrit: & 58,06 & 19,26 & $\overline{8,8}$ & 2,22 & 3,14 & 4,17 & 1,2 & 0,61 & 0,16 & 0,15 & 2,23 & \\
\hline LA-02 & Civita Castellana, I & Ignimbrita, traquiandesita & 52,52 & 16,41 & 6,34 & 0,58 & 7,54 & 3,67 & 1,73 & 0,51 & 0,1 & 0,17 & 10,43 & 99 \\
\hline TO -03 & Pitigliano, I & Pumita, traquita & 55,35 & 19,2 & 8,25 & 2,35 & 2,95 & 4 & 1,15 & 0,54 & 0,13 & 0,17 & 6 & \\
\hline GC-05 & Arguineguİn, E & Ignimbrita, traquita & 58,8 & 16,33 & 5,34 & 5,9 & 0,4 & 3,79 & 0,75 & 0,77 & 0,3 & 0,03 & 7,6 & $\overline{99}$ \\
\hline TE-03 & Chimiche, E & Pumita, traquita & \begin{tabular}{|l|}
57,63 \\
\end{tabular} & 18,4 & $\overline{5,41}$ & 6,58 & 0,57 & 3,29 & 0,57 & 0,47 & 0,25 & 0,03 & 6,8 & $\overline{99}$ \\
\hline ES-03 & Vera, E & Obsidiana, lamproita & 58,42 & 10,8 & 8,35 & 1,5 & 2,56 & 5,8 & 8,68 & 1,4 & 0,09 & 0,76 & 1,15 & \\
\hline
\end{tabular}




\subsection{Caracterización químico-mineralógica de las rocas volcá- nicas empleadas como materia prima del cemento}

En la totalidad de las muestras de roca se realizó una lámina delgada para su estudio con el microscopio petrográfico, algunas fueron seleccionadas para el estudio de la química mineral y del vidrio mediante microsonda electrónica. Todas las muestras fueron pulverizadas en mortero de acero y se procedió a la caracterización química de sus componentes mayoritarios mediante espectrometría de fluorescencia de rayos $\mathrm{X}$ (FRX), método considerado internacionalmente como un estándar de calidad para este tipo de análisis. Todas las muestras fueron deshidratadas a $130{ }^{\circ} \mathrm{C}$ en recipientes de pyrex durante 48 horas antes de cualquier otra manipulación. Como norma se realizó el análisis por duplicado en dos de los laboratorios implicados (Serveis Científico-Tècnics de la Universitat de Barcelona, SCT-UB y DIGITA dell'Università di Cagliari, UCA), en algunos casos en tres (BRGM Orléans, Francia). En ambos casos se empleó un espectrómetro secuencial de rayos X Phillips PW1400. Los métodos empleados han diferido ligeramente.

En SCT-UB los mayores fueron determinados sobre perla de tetraborato de litio (dilución 1/20; perlas obtenidas por triplicado en crisol y plato colector de $\mathrm{Pt}$, usando LiI como corrector de viscosidad) y fueron determinados por duplicado (original y réplica, la tercera perla realizada con función de limpieza; además el crisol fue regularmente sometido a limpieza con ácido nítrico). El espectrómetro fue calibrado empleando un juego de más de 60 patrones internacionales (véase los detalles en http://www.sct.ub.es/serveis/01030202/udoc_ 003.htm). Un juego de patrones internacionales suministrados por el Geologic Survey of Japan fue empleado como control interno de la calidad de los resultados. El Na fue determinado también por EAA sobre patrones internos como control, aunque los problemas de determinación de este elemento en contenidos bajos (inferiores al $2.5 \%$ de $\mathrm{Na}_{2} \mathrm{O}$ ) como norma no se han presentado, dada la naturaleza alcalina de las muestras estudiadas.

En UCA los elementos se determinaron por duplicado sobre pastillas prensadas (6 gr de muestra aglutinadas con alcohol polivinílico y prensadas en una cápsula de $\mathrm{Al}$ sobre una base de ácido bórico). Se empleó el método (5), y a diferencia del caso precedente se empleó en el tratamiento de los datos brutos del espectrómetro un software propio (Garbarino, no publicado) que permite corregir los fenómenos de interferencia de matriz de los elementos mayoritarios determinados en pastilla. Los resultados obtenidos en ambos casos son perfectamente comparables y las diferencias menores al error experimental en FRX.

Se ha calculado la pérdida al fuego (LOI) de 1 gr de muestra mediante calcinación en un crisol cerámico durante $2 \mathrm{~h}$ a $950{ }^{\circ} \mathrm{C}$ en un horno oxidante, por duplicado (valor promedio), tomando este valor como una indicación del contenido en volátiles.

La geoquímica de elementos mayores ha sido empleada para clasificar las muestras siguiendo las indicaciones de la IUGS (6). Por lo tanto, los valores recalculados sobre base anhidra han sido proyectados en el diagrama TAS (sílice contra sumatorio de los alcalinos) y la norma CIPW clásica de petrología ígnea se ha empleado para discriminar entre rocas que se proyectan en el mismo campo. Las normas han sido calculadas con el programa newpet (7). Todos esos datos han sido cruzados con la petrografía considerando con particular atención la posible presencia de alteración de las rocas y el eventual posible desacuerdo entre las clasificaciones químicas y petrográficas.

La química de las fases minerales presentes en las rocas y de los vidrios fueron determinadas mediante el empleo de una microsonda electrónica CAMECA Camebax SX-50 en los laboratorios del BRGM (Orléans) mediante protocolos analíticos análogos a los descritos por (8). De igual modo se procedió con el estudio de las probetas de los morteros que serán comentadas más adelante.

La composición mineralógica de las rocas fue determinada mediante el empleo de un difractómetro de rayos X SIEMENS D-500 (DRX). Se obtuvo los espectros de difracción en el rango $4-70^{\circ} 2 \Theta$ con un salto de barrido $\mathrm{h} 0.05^{\circ} 2 \Theta$ p y un tiempo de cuenta de $5 \mathrm{~s}$, usando radiación $\mathrm{CuK} \alpha$, unas condiciones de tubo de $40 \mathrm{KV}$ y $28 \mathrm{~mA}$ y un monocromador de grafito.

\subsection{Formulación de los cementos y morteros}

El procedimiento de fabricación de un cemento geopolimérico que no contiene cemento portland consiste en la preparación de una mezcla reactiva en la que los componentes expresados en materia seca son:

- reactivo 1: 100 partes en peso de un óxido aluminosilicático $\left[\mathrm{Si}_{2} \mathrm{O}_{5}, \mathrm{Al}_{2} \mathrm{O}_{2}\right]_{9}\left[\mathrm{Si}_{2} \mathrm{O}_{5}, \mathrm{Al}_{2}(\mathrm{OH})_{3}\right]$, teniendo el catión $\mathrm{Al}$ en coordinación mixta (IV-V), para expresarlo en manera simplificada, $\left(\mathrm{Si}_{2} \mathrm{O}_{5}, \mathrm{Al}_{2} \mathrm{O}_{2}\right)_{(\mathrm{IV}-\mathrm{V})}$.

- reactivo 2: 30-55 partes de silicato de sodio y/o potasio en el que la relación $\mathrm{M}_{2} \mathrm{O} / \mathrm{SiO}_{2}$ está comprendida entre 0.5 y 0.8 , donde $\mathrm{M}$ designa $\mathrm{Na}$ y/o K o la mezcla $\mathrm{Na}+\mathrm{K}$.

- reactivo 3: 80-110 partes de silicato básico, en estado vítreo, compuesto en parte por gehlinita, akermanita y wollastonita.

- Reactivo 4: 150-250 partes de aluminosilicato alcalino conteniendo al menos un $5 \%$ en peso de $\left(\mathrm{Na}_{2} \mathrm{O}+\mathrm{K}_{2} \mathrm{O}\right)$, preferentemente con un mínimo de un $8 \%$ en peso

Posteriormente se hace endurecer la citada mezcla añadiendo agua.

El reactivo 1, el óxido alumínico silicatado de fórmula simplificada $\left(\mathrm{Si}_{2} \mathrm{O}_{5}, \mathrm{Al}_{2} \mathrm{O}_{2}\right)_{(\mathrm{IV}-\mathrm{V})}$ se obtiene por calcinación de un material caolinítico a una temperatura inferior a $1000^{\circ} \mathrm{C}$, en nuestro caso típicamente a $750{ }^{\circ} \mathrm{C}$. El reactivo 2 es el silicato de sodio y/o de potasio, en polvo, por ejemplo el disilicato de potasio $\mathrm{K}_{2}\left(\mathrm{H}_{3} \mathrm{SiO}_{4}\right)_{2}$, o una mezcla de silicato y $\mathrm{NaOH} / \mathrm{KOH}$ solido. Se puede de esta manera utilizar el silicato alcalino en forma de una solución de silicato de potasio conteniendo $20-30 \%$ en peso de $\mathrm{SiO}_{2}, 20-26 \%$ en peso de $\mathrm{K}_{2} \mathrm{O}, \mathrm{y} 50-55 \%$ en peso de agua.

El reactivo 3 es un silicato de calcio débilmente cálcico, es decir que tiene una relación atómica $\mathrm{Ca} / \mathrm{Si}$ inferior a 1 . Se obtiene utilizando como materia prima un silicato de calcio básico, es decir teniendo $\mathrm{Ca} / \mathrm{Si}$ igual o superior a 1 esencialmente caracterizado por su aptitud para generar, bajo la acción de un ataque alcalino, la formación de silicato de calcio débilmente básico, es decir teniendo una relación atómica $\mathrm{Ca} / \mathrm{Si}$ inferior a uno y, preferentemente, próxima a 0.5.

El reactivo 4 es un material geológico (previamente descrito), químicamente caracterizado como un aluminosilicato alcalino, en el que la relación atómica Si:Al está comprendida entre 2.5 y 5, la relación molar $\mathrm{M}_{2} \mathrm{O}: \mathrm{Al}_{2} \mathrm{O}_{3}$ está comprendida entre 
0.7 y 1.1 y la relación molar $\left(\mathrm{M}_{2} \mathrm{O}+\mathrm{CaO}\right): \mathrm{Al}_{2} \mathrm{O}_{3}$ oscila entre 0.8 y 1.6. En general este material es calcinado a una temperatura inferior a $850^{\circ} \mathrm{C}$, aunque algunas rocas de las utilizadas han reaccionado igualmente de una manera satisfactoria en términos de resistencia mecánica sin calcinar (véase discusión y conclusiones en el apartado 6); en cualquier caso, la calcinación presenta como ventaja la disminución de la cantidad de agua que hay que añadir a la mezcla para obtener una buena fluidez en el mortero.

La mezcla de los reactivos 1, 2 y 3 se dosifica con agua en las proporciones en peso 30:25:27:31 (donde 31 corresponde al agua, $\mathrm{y}$ en total tenemos 113 partes en peso) lo que le confiere una relación molar $\mathrm{Ca}^{++} /\left(\mathrm{Si}_{2} \mathrm{O}_{5}, \mathrm{Al}_{2} \mathrm{O}_{2}\right)_{(\mathrm{IV}-\mathrm{V})}$ superior a $1 \mathrm{y}$ una relación $\left.\mathrm{Na}^{+}, \mathrm{K}^{+}, \mathrm{Ca}^{++}\right) /\left(\mathrm{Si}_{2} \mathrm{O}_{5}, \mathrm{Al}_{2} \mathrm{O}_{2}\right)_{(\mathrm{IV}-\mathrm{V})}$ superior a 1,5. Después del endurecimiento tenemos el cemento base geopolimérico que está constituido por dos fases distintas, una que consideramos vítrea que posee un espectro de resonancia MAS-NMR ${ }^{29} \mathrm{Si}$ (véase apartado 4) con una banda comprendida entre -85 y $-89 \mathrm{ppm}$, y un espectro MAS-NMR ${ }^{27} \mathrm{Al}$ con una resonancia en 54-58 ppm; y una segunda fase cristalina que corresponde a un aluminosilicato cristalino y presenta un espectro MASNMR ${ }^{29} \mathrm{Si}$ con una banda de resonancia comprendida entre -90 ppm y -115 ppm, y un espectro MAS-NMR ${ }^{27} \mathrm{Al}$ con una resonancia en $57 \mathrm{ppm}$.

Finalmente, se ha tomado 113 partes en peso de cemento base geopolimérico y se les han añadido 50 partes en peso de aluminosilicatos alcalinos naturales (roca volcánica, previamente sometida a molienda hasta granulometría media de 8 micras), la experiencia se ha realizado tanto sobre material crudo como con la misma roca después de 3 horas de calcinación en horno a $800{ }^{\circ} \mathrm{C}$. El cemento así obtenido, que llamamos pasta, se ha vertido sin adición de carga en un molde y se ha dejado endurecer a temperatura ambiente. La principal diferencia entre este cemento y el previamente desarrollado a partir de reactivos químicos en el laboratorio es la aportación de este último reactivo natural que substituye cantidades comparables del reactivo 2.

Por lo que se refiere a los morteros, una vez estudiados los resultados de la resistencia a la compresión de la familia de cementos desarrollados con la roca natural, se procedió a realizar tests sobre morteros desarrollados en escala semiindustrial sobre la muestra SA07, seleccionada en función de todos los parámetros estudiados en el proyecto. Se trata de una roca piroclástica del tipo ash-flow, pobremente soldada, correspondiente a la Formación Paringianu (9) del SO de la isla de Cerdeña, que aflora ampliamente en óptimas condiciones para su explotación; esta unidad fue totalmente remuestreada. Los morteros se realizaron con el reactivo volcánico natural sometido previamente a calcinación a $800{ }^{\circ} \mathrm{C}$. La mezcla arenacemento se realizó según el procedimiento y norma europea, es decir: los reactivos en polvo 1, 3 y 4 se homogeneizaron durante 30 segundos con arena normalizada (ISO), después se añadió el reactivo 2 líquido y el agua mezclando durante 270 segundos, y se finalizó el proceso a gran velocidad durante 60 segundos. Esta mezcla se vertió en moldes normalizados (DIN 1164). El mortero contiene 450 partes de arena por 190 parte de cemento más agua. Con el fin de obtener una buena laborabilidad del mortero, el cemento geopolimérico se modificó y realizó con la siguiente mezcla (en peso, en el orden en que han sido numerados, el último valor corresponde al agua) de reactivos: 23:23.4:20.3:56.7:29, más un 1\% del peso del cemento correspondiente a un superplastificante (sulfonato de naftaleno).

El mortero así realizado endurece a los 30 minutos. Permanece 24 horas en el molde, siendo extraído de él y dejado inmerso en agua periodos de 7 y 28 días de cara a la posterior realización de las pruebas de resistencia mecánica.

\subsection{Tests desarrollados en los cementos y morteros}

Han consistido principalmente en tres tipos de caracterizaciones: estudio de la resistencia mecánica (cemento y morteros), caracterización de los cementos $\left({ }^{29} \mathrm{Si},{ }^{27} \mathrm{Al}\right)$ mediante resonancia magnética nuclear (RMN) de alta resolución (magic-angle spinning, MAS-NMR) y test de lixiviación de los morteros en ambiente ácido. En esta nota se hace referencia sólo a los dos primeros, la resistencia mecánica en tanto permite caracterizar las propiedades físicas y compararlo a cementos tradicionales de base carbonatada, y la RMN en tanto permite establecer, comparando con los productos naturales de partida las variaciones estructurales de los elementos que permiten establecer la química del proceso de formación del cemento y que a fin de cuentas son las que le confieren sus principales características físico-mecánicas. Los estudios de resistencia mecánica se han realizado por lo que se refiere al cemento en los laboratorios de Cordi-Géopolimère en Saint Quentin (Francia); mientras que los estudios estandarizados de los morteros empleando las muestras y los protocolos normalizados correspondientes y los estudios de RMN fueron contratados a laboratorios externos (respectivamente: a Cementi Buzzi, Italia; y al Lab. MAS-NMR de la Université de Namur, Belgica, resp. Z.Gabelica). Los métodos empleados son suficientemente conocidos (norma europea en el caso de los cementos, véase 3.2.; véase p.e. (10) en el caso de la MAS-NMR).

\section{CONTROLES SOBRE LA MODIFICACIÓN DE LAS MA- TERIAS PRIMAS DURANTE EL PROCESO DE FABRI- CACION DEL CEMENTO GEOPOLIMÉRICO.}

El campo de los geopolímeros ha adquirido un notable desarrollo en los últimos 50 años, tanto científico como industrial (11). El estudio de los productos geopoliméricos puede ser afrontado al menos desde dos puntos de vista diferentes: uno de carácter más químico y otro más petrológico-mineralógico. El estudio de los tectosilicatos es un tema de estudio preferente en las ciencias de la tierra desde el inicio de la cristalografía geométrica, hace por tanto más de 150 años. Sin embargo, dentro de los tectosilicatos el grupo de las zeolitas recibió tradicionalmente poca atención, debido principalmente al carácter microcristalino de la mayoría de las especies minerales implicadas. Por este motivo, ha sido el mundo de la ingeniería química el que guió los primeros estudios sobre síntesis de zeolitas, desde los trabajos pioneros de Barrer en los años 40 (véase p.e. una revisión del tema en (12). Por otra parte, es comprensible que los esfuerzos en este campo, que han partido de unos usos industriales específicos (en los campos del filtrado molecular, la catálisis, el intercambio iónico, entre otros) se hayan concentrado en el desarrollo (y en la optimización económica de éste) de zeolitas sintéticas. Esto se debe a que estos usos industriales específicos requieren zeolitas de gran pureza, muchas de las cuales son metaestables en los ambientes geológicos naturales (véase por ej. 12, 13, 14) y por lo tanto de 
vida efímera en el registro geológico, y a que los yacimientos minerales, a medida que se han ido conociendo con un cierto detalle se han demostrado en general bi- o poliminerálicos, siendo la separación de las especies minerales implicadas muy difícil en términos técnicos y en general impracticable en términos económicos.

En general, los procedimientos de síntesis a partir de los que se desarrolló la patente original que originó el estudio que nos ocupa $(2,4)$ son bien conocidos desde hace algunos años, en el campo de la producción de productos zeolíticos inconsolidados o friables. Hay que entender por tanto que el principal interés de la patente radica en la producción de un cemento zeolítico compacto, que se pueda dosificar y aplicar en morteros. Por otra parte este cemento presenta respecto a un cemento carbonatado, entre otras ventajas, una notable resistencia al ataque ácido que le confiere un interés específico en el campo del encapsulado definitivo de residuos tóxicos.

Así, existen numerosas técnicas (véase p.e. 12) y algunas patentes que, con variaciones de detalle, producen una zeolita sintética partiendo de la activación de una fase arcillosa y su posterior mezcla con un componente silícico-alcalino (véase p.e. 12, 13, 15). El empleo de un reactivo arcilloso tiene por misión proporcionar el aluminio requerido (y, según el tipo y pureza de la arcilla, parte del silicio) para la síntesis de la zeolita. Esta fase arcillosa puede ser activada mediante diferentes procedimientos; en el caso de la activación térmica diferentes técnicas (DRX, estudio del espectro de absorción de banda infrarroja, MNR, análisis termogravimétrico y termodiferencial, entre otras, 10,11, 13, 16, 17) permiten constatar que la metakaolinita obtenida por calcinación a temperaturas superiores a $600-650{ }^{\circ} \mathrm{C}$ esencialmente presenta la pérdida de la estructura tetraédrica (tetraedros de sílice) hojosa propia del filosilicato y su substitución por un producto que ha sido definido por diferentes autores como un gel o una fase amorfa reactiva. La técnica del MAS-MNR destaca por su precisión, ya que permite caracterizar independientemente el estado de coordinación propio de los elementos que constituyen la parte esencial de la estructura (Si y $\mathrm{Al}$ ) y por lo tanto registrar el momento en el que acontecen los principales cambios durante la fabricación del cemento. En general, podemos resumir que en los silicatos (incluyendo las zeolitas) los espectros MAS-MNR de ${ }^{27} \mathrm{Al}$ y ${ }^{29} \mathrm{Si}$ se caracterizan por precisos picos de resonancia correspondientes a estados de coordinación de estos elementos que reflejan una estructura ordenada (10). El proceso de activación térmica del precursor kaolinítico conduce a la destrucción de la estructura tetraédrica hojosa (16) caracterizada por picos de resonancia del ${ }^{27} \mathrm{Al}$ alrededor de 0 y -2.5 ppm correspondientes a coordinación octaédrica y del ${ }^{29} \mathrm{Si}$ alrededor de -90 ppm, correspondientes a su vez a coordinación tedraédrica. Esta destrucción de la estructura y la consiguiente formación del metakaolín se produce a partir de los $600{ }^{\circ} \mathrm{C}$ hasta los $900{ }^{\circ} \mathrm{C}$. El resultado es la aparición de otros picos de resonancia del ${ }^{27} \mathrm{Al}$ alrededor de 23 ppm (coordinación 5, véase (18)) y 57.4 ppm (coordinación tetraédrica), y el desplazamiento hacia valores alrededor de -100 ppm y un gran ensanchamiento del pico de resonancia correspondiente a ${ }^{29} \mathrm{Si}$. En el procedimiento empleado la génesis del metakaolín se realiza en un horno vertical a $750{ }^{\circ} \mathrm{C}$; a temperaturas mayores se produce una reestructuración total que da lugar a la génesis de mullita (16). La génesis de una fase zeolítica cristalina conlleva la reordenación tetraédrica del ${ }^{29} \mathrm{Si}$ conducente a la formación de un estrecho pico de resonancia próximo a los valores iniciales (entre -80 y -90 ppm) y la reordenación igualmente tetraédrica del ${ }^{27} \mathrm{Al}$ con la formación de un único pico en el espectro de resonancia alrededor de 57 ppm (17).

La técnica MAS-MNR ha sido empleada para comprobar la calidad del kaolín activado térmicamente empleado en la reacción $\left({ }^{29} \mathrm{Si},{ }^{27} \mathrm{Al}\right)$, con resultados plenamente coincidentes con los disponibles en la bibliografía $(16,17)$, para comprobar las características de la base geopolimérica producida en la reacción entre dicho kaolín activado y la escoria de alto horno $\left.{ }^{(29} \mathrm{Si},{ }^{27} \mathrm{Al}\right)$ y para estudiar las características de los cementos producidos a partir del reactivo volcánico natural calcinado a $800{ }^{\circ} \mathrm{C}\left({ }^{29} \mathrm{Si}\right)$. Los espectros ${ }^{29} \mathrm{Si}$ MAS-MNR de estos cementos (fig. 2) muestran como característica más notable una amplia franja de respuesta en valores comprendidos entre -80 y -110 ppm, consistente en un ancho pico variable de cemento a cemento, y que sólo localmente muestra picos individualizados (p.e., el cemento SA01 presenta un marcado pico en valores próximos a $107 \mathrm{ppm}$, atribuíble a la presencia de feldespatos alcalinos (10). La interpretación de estos espectros no es sencilla; si se considera que corresponde a una única fase implicaría un elevado grado de desorden y una muy baja cristalinidad. Por el contrario, si se compara con el espectro ${ }^{29} \mathrm{Si}$ MAS-MNR correspondiente a base geopolimérica (que proporciona un pico ancho en el tramo del espectro -84 a -90 ppm) podemos pensar que de hecho estamos ante la superposición de los espectros de diferentes componentes del cemento, por un lado
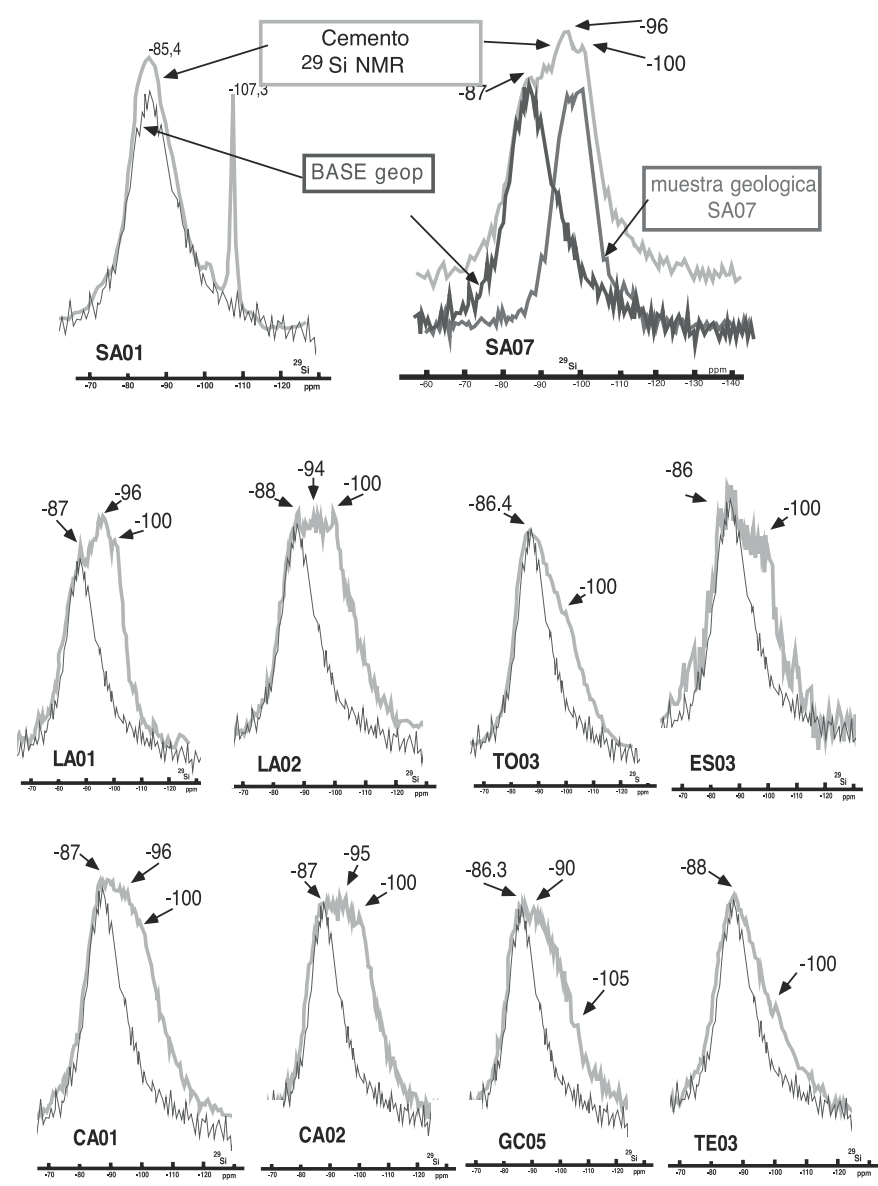

Fig. 2. Espectros de resonancia ${ }^{29} \mathrm{Si}$ MAS-MNR de los cementos desarrollados con la roca volcánica natural calcinada a $800{ }^{\circ} \mathrm{C}$ (trazo grueso) comparados con el equivalente de la base geopolimérica (trazo fino) (véase explicación en el texto). 
la base geopolimérica y por otra el producto volcánico calcinado. La substracción de la base geopolimérica a los espectros proporciona espectros comparables a los de algunas zeolitas naturales (10), aunque claramente desdibujados (p.e., con los picos de resonancia ensanchados o ligeramente desplazados), un factor atribuible a la calcinación de la muestra. Como no disponemos de la totalidad de los espectros correspondientes a las muestras geológicas previas a la calcinación es difícil extraer más conclusiones en este sentido.

En todo caso, tal como queda patente en el título de este trabajo, nuestro principal objetivo es demostrar como técnicas mucho más accesibles y económicas (DRX, FRX) permiten una eficaz interpretación de los resultados físico-mecánicos obtenidos de las probetas realizadas a partir de los cementos producidos, dando criterios de tipo práctico y fácil aplicación industrial.

\section{DISCUSIÓN DE LOS RESULTADOS OBTENIDOS EN LA FASE DE PROSPECCIÓN GEOLÓGICA E INTER- PRETACIÓN DE LOS ENSAYOS REALIZADOS SOBRE CEMENTOS PREINDUSTRIALES}

La tabla 1 y la fig. 3 muestran con claridad que las muestras estudiadas corresponden a rocas diferenciadas, principalmente a las rocas que ocupan el campo de las riolitas y las traquitas (en menor medida, dacitas) en el diagrama de clasificación TAS (5); dentro de cada uno de los campos considerados ocupan el sector superior que corresponde a las rocas más alcalinas den- tro de cada grupo. Este hecho no es sorprendente, si se tiene en cuenta que el muestreo es el resultado de una minuciosa selección sobre las fuentes documentales y que los resultados analíticos necesariamente debían corroborar en gran medida los disponibles, a pesar de que lógicamente la elección de nuestros puntos de muestreo no se ha desarrollado sobre los puntos conocidos (muy frecuentemente no referidos a rocas piroclásticas, sino a rocas lávicas masivas) sino sobre formaciones favorables a nuestros objetivos, de carácter piroclástico y dentro de éstas pobre o nulamente soldadas por fenómenos sin-post-eruptivos. La marcada ausencia de rocas de composición fonolítica se debe principalmente a que en el ámbito geográfico objeto de nuestro estudio las potenciales rocas fonolíticas objeto de nuestro interés presentan en general una afinidad marcadamente sódica, motivo por el que se descartó su estudio y muestreo.

Como singularidad hay que señalar que la muestra ES-03 procedente del SE peninsular, aunque aparezca proyectada en el campo de las traquitas/traquidacitas junto al límite de las traquiandesitas, en realidad es una muestra lamproítica que ha sido escogida para contrastar si, a pesar de su elevado contenido en elementos alcalinos presenta escasas aptitudes para la fabricación del cemento, debido a su elevado contenido en $\mathrm{Mg}$, elemento considerado a priori como penalizante en el desarrollo de la fórmula antes expuesta. De hecho este tipo de roca, muy infrecuente en la naturaleza, sólo podría ser objeto de interés económico en unas pocas regiones del globo y, en el caso europeo, en el SE de la Península Ibérica. Los resultados, óptimos como se puede apreciar (tabla 2), indican que en la

TABLA 2.- RELACIONES ENTRE LOS PRINCIPALES ÓXIDOS (EXPRESADOS EN \%), CONTENIDO EN VOLÁTILES Y COMPONENTES PRINCIPALES (MINERALES Y, EN SU CASO, VIDRIO) DE LAS MUESTRAS DE LA TABLA 1. LOS COMPONENTES EXPRESADOS ENTRE PARÉNTESIS APARECEN COMO TRAZAS O EN TODO CASO EN CANTIDADES SENSIBLEMENTE INFERIORES AL RESTO. R INDICA LA RESISTENCIA A LA COMPRESIÓN A LOS 28 DÍAS, DE LOS CEMENTOS DESARROLLADOS A PARTIR DE ESTAS MUESTRAS, SIN CARGAS ADICIONALES DE ÁRIDOS O ARENAS (VÉASE EXPLICACIÓN EN EL TEXTO). LOS DATOS DE R PROCEDEN DE (1).

\begin{tabular}{|l|r|r|r|r|r|r|c|}
\hline Muestra & $\mathrm{R}$ Natural & $\mathrm{Na}_{2} \mathrm{O}+\mathrm{K}_{2} \mathrm{O}$ & $\mathrm{SiO}_{2} / \mathrm{Al}_{2} \mathrm{O}_{3}$ & $\mathrm{~K}_{2} \mathrm{O} / \mathrm{Na}_{2} \mathrm{O}$ & $\mathrm{H}_{2} \mathrm{O}$ & $\mathrm{R} 800^{\circ} \mathrm{C}$ & $\begin{array}{c}\text { Componentes determ inad os por DRX } \\
\text { (muestra natural) }\end{array}$ \\
\hline ES-03 & 50 & 10,1 & 5,4 & 5,6 & 1,15 & 50 & Vidrio (feldespato) \\
\hline LA-01 & 48 & 11,3 & 3,0 & 4,0 & 2,23 & 53 & Feldespato > analcima (illita) \\
\hline SA-01 & 48 & 11,2 & 4,9 & 15,1 & 0,96 & 35 & Feldespato > cuarzo \\
\hline SA-07 & 50 & 9,3 & 5,4 & 1,1 & 0,92 & 45 & Feldespato + tridimita \\
\hline CA-02 & 35 & 10,0 & 3,2 & 3,7 & 6,75 & 47 & Phillipsita + feldespato > illita (vidrio) \\
\hline TE-05 & 33 & 12,2 & 3,6 & 0,9 & 7,60 & 50 & Phillipsita (otras zeolitas) \\
\hline TO-03 & 32 & 12,9 & 3,1 & 0,8 & 6,80 & 51 & Vidrio + phillipsita \\
\hline LA-02 & 32 & 11,3 & 2,9 & 3,5 & 6,00 & 51 & Feldespato + analcima > illita (vidrio) \\
CA-01 & 31 & 7,8 & 3,2 & 10,9 & 10,43 & 42 & Phillipsita + chabazita (vidrio) \\
\hline
\end{tabular}

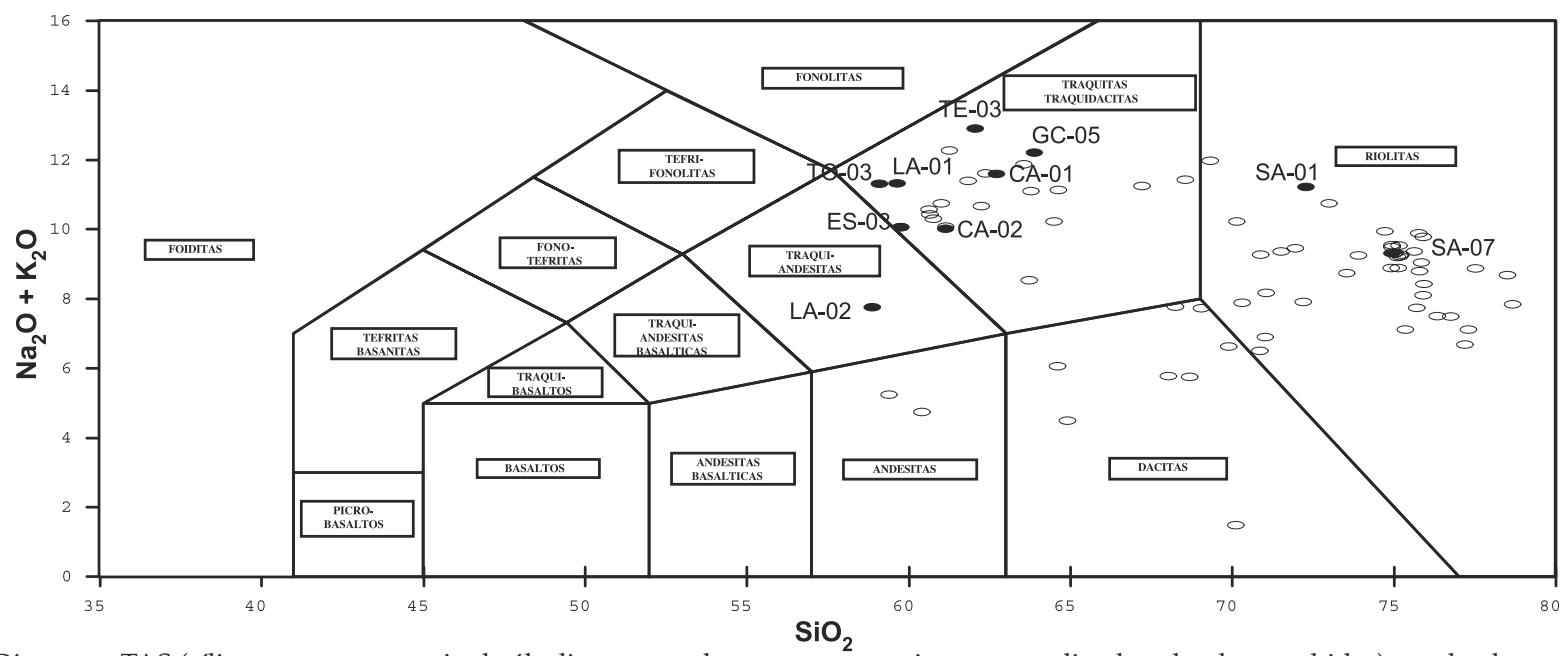

Fig. 3.- Diagrama TAS (sílice contra sumatorio de álcalis, expresado en tantos por ciento normalizado sobre base anhidra) empleado para la clasificación de los tipos litológicos analizados en la fase de prospección inicial del proyecto GEOCISTEM. Se han resaltado las muestras empleadas en la fabricación de cementos de base silicatada (véase explicación en el texto). 
química de la reacción actúan otros parámetros que deben ser controlados (véase apartado 6). Por su parte, la muestra SA-01 también fue incluida a efectos de control y comprensión de la química del cemento, ya que de hecho no es una riolita como pudiera deducirse de su posición en el seno del diagrama TAS, sino que se trata de una andesita sometida a un intenso proceso de alteración potásica durante el cual ha sufrido igualmente un balance marcadamente positivo por lo que se refiere al silicio.

Si atendemos a algunos de los otros parámetros clásicos de clasificación de rocas ígneas, la mayor parte de las rocas destacan por un carácter peralumínico (fig. 4), pero también hay rocas metalumínicas y otras peralcalinas; no parece por lo tanto que las variaciones entre la relación molar alúmina contra sumatorio de óxidos alcalinos a priori resulte significativa en la población analizada. Hay que tener en cuenta, de todos modos, que el significado petrológico de estos parámetros geoquímicos aún siendo significativo de los equilibrios molares en la roca no es directamente transferible a la síntesis zeolítica que nos ocupa; sencillamente implica que es más probable la formación de unas especies zeolíticas que otras a partir de un precursor de una composición dada (12). Por otra parte, si atendemos al diagrama clásico de clasificación de las rocas volcánicas en función de su contenido en potasio (6) recogido en la fig. 5, queda claro que la mayor parte de las rocas, y en concreto las empleadas en las pruebas de fabricación de cemento de base silicatada, quedan en general por encima del contenido máximo de las rocas consideradas "altas en potasio", cumpliendo por lo tanto con el requisito básico (1) expresado para estas rocas como fuente substitutiva de este elemento en la formulación (2) del cemento.

Dado que la prospección geológica culminó plenamente sus objetivos con la obtención de una amplia gama de rocas naturales potencialmente utilizables en la fabricación de un cemento silicatado, se seleccionó un juego de 10 muestras (tablas 1 y 2) que fueron empleadas en el desarrollo de los cementos; el lector interesado hallará información complementaria sobre estas y otras pruebas técnicas como la retracción hidráulica, resistencia al sulfato, resistencia a la corrosión ácida, etc., en (3). En resumen, del conjunto de resultados analizados se puede deducir que, con diferentes grados, la práctica totalidad de productos empleados presentan un uso potencial en el de-

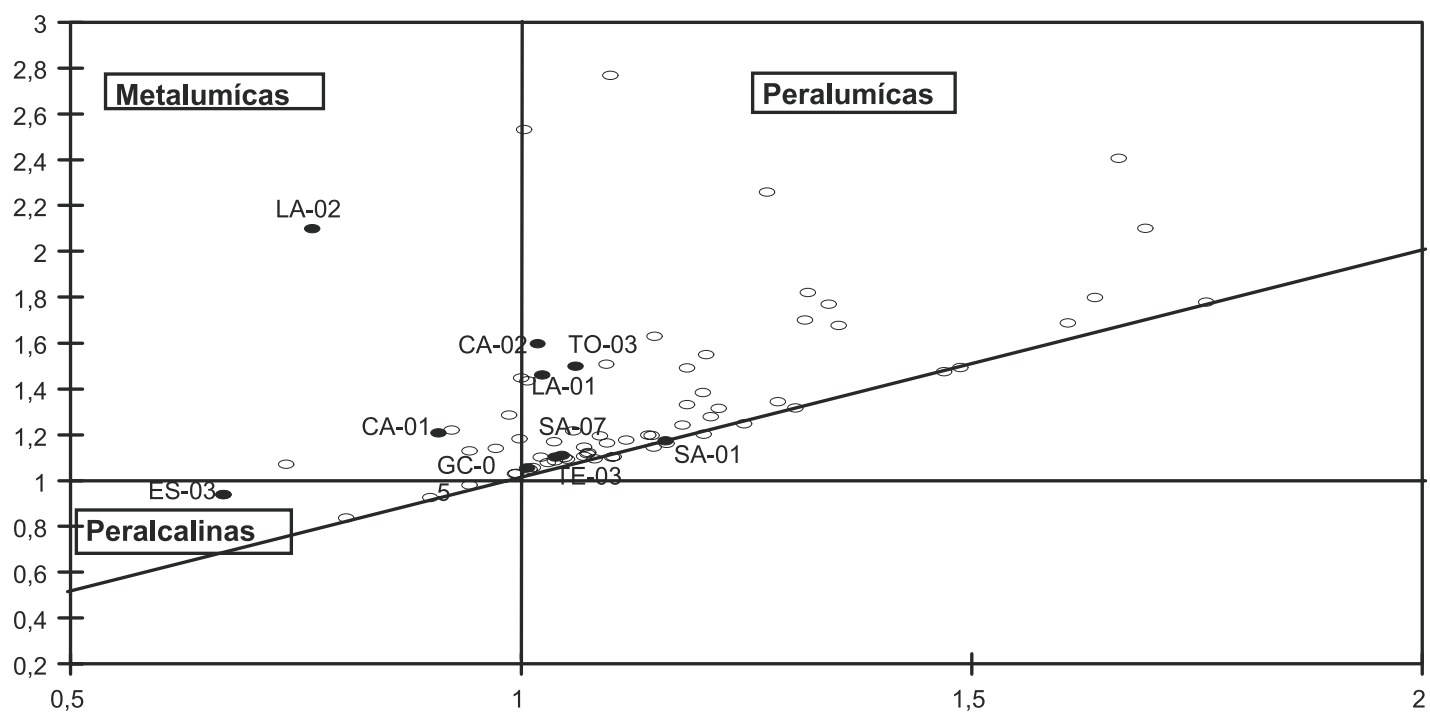

Fig. 4.- Diagrama de clasificación de las rocas estudiadas en la fig. 2 atendiendo a la relación molar entre alúmina y el sumatorio de álcalis (véase explicación en el texto).

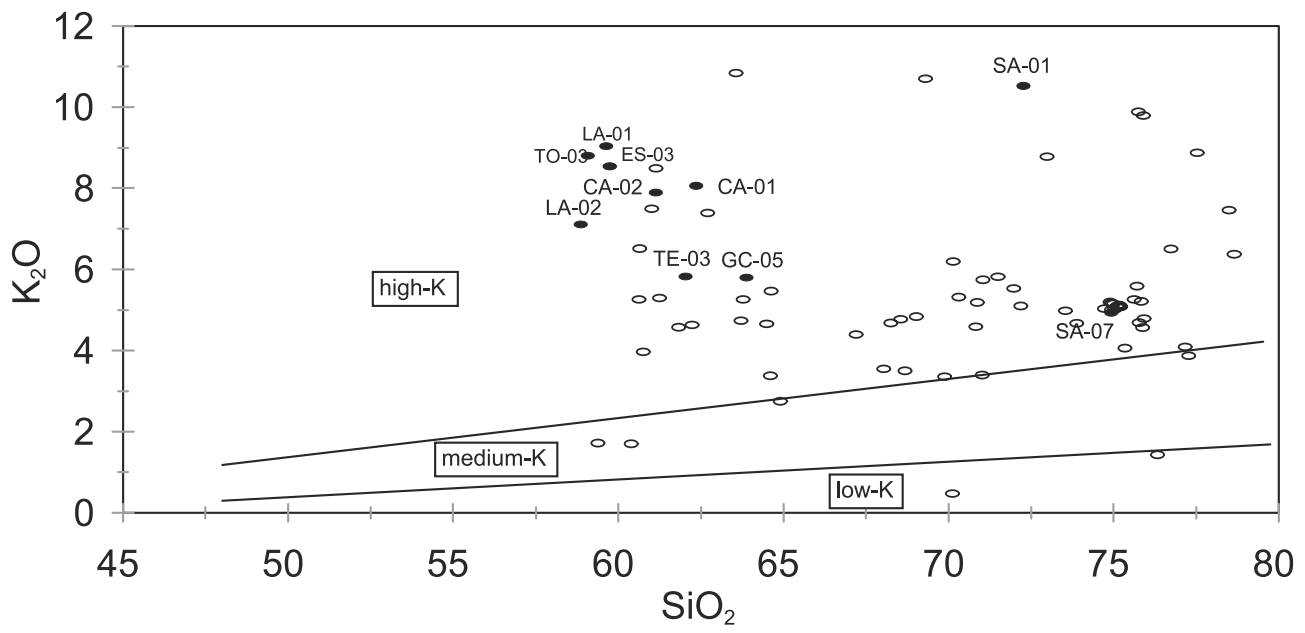

Fig. 5.- Diagrama de clasificación de las rocas volcánicas estudiadas en la figura 2 atendiendo a su contenido en potasio (véase explicación en el texto). Los valores por encima del $4.5 \%$ de $\mathrm{K}_{2} \mathrm{O}$ no aparecen contemplados en el diagrama original 
sarrollo de cementos comparables a los obtenidos empleando reactivos químicos caros (en particular silicato de potasio) si bien en la formulación práctica no se llegó a eliminar totalmente su empleo como catalizador de las reacciones generadoras del cemento $(1,3)$.

Por lo que se refiere a los resultados del mortero desarrollado, se midieron la resistencia a la compresión y a la flexión a las 24 horas a la salida del molde, y a los 7 y 28 días de permanencia en el agua, dando valores respectivamente de 10.5, 34 y $42.5 \mathrm{~N} / \mathrm{mm} 2$ en el caso de la compresión y 2.8, 5.8 y 7.4 $\mathrm{N} / \mathrm{mm} 2$ en el caso de la flexión. Estos resultados entraban dentro de los aceptables en las premisas de partida, habiendo sido comparados durante las experiencias con los obtenidos por otros morteros semejantes realizados con un cemento hidráulico de base portland, concretamente el CEM I 42.5 R de Cementi Buzzi.

\section{CONCLUSIONES DE CARÁCTER TÉCNICO-ECONÓ- MICO: COMPARACIÓN DE LOS RESULTADOS OB- TENIDOS A PARTIR DE MATERIA PRIMA CRUDA $Y$ CALCINADA.}

Un segundo tipo de resultados del proyecto está relacionado con la metodología de empleo del producto natural, y el costo en términos energéticos de su proceso de incorporación a la formulación base del cemento. Se trata por tanto de conclusiones de tipo técnico-económico que pueden permitir la aplicación industrial de los cementos desarrollados

\subsection{Metodología de empleo de la roca volcánica.}

Los socios industriales de este estudio partieron de un proceso de vitrificación y posterior disgregación del suministrador de alcalinos, en concreto la roca volcánica. Esto implica fusiones a temperaturas superiores a $1000{ }^{\circ} \mathrm{C}$, o el empleo de aditivos fundentes en principio no contemplados en la formulación del cemento. Este tipo de proceso es más adecuado en el caso de la industria cerámica (p.e., en la producción de gres porcelánico) donde el valor añadido del producto final puede compensar los costes energéticos (e incluso los de la adición de un fundente). Desde el punto de vista geológico, es un tanto recurrente tomar un producto vítreo fragmentario, vitrificarlo de nuevo, disgregarlo de nuevo, y añadirlo a la formulación; las únicas ventajas aparentes de este procedimiento radican por una parte en el proceso de homogeneización obtenido en la materia prima y en la eliminación de productos cristalinos de la masa del vidrio. Desde el punto de vista estrictamente científico, creemos que en este trabajo se aportan además suficientes argumentos para poder concluir que el hecho de que un vidrio natural pueda ser potencialmente más reactivo dependerá esencialmente no de su carácter intrínseco de vidrio sino de su estado de polimerización y del estado de coordinación de los principales elementos implicados en la formulación del cemento, un campo aún pobremente conocido en la actualidad.

La homogeneidad del producto en principio es inherente a la elección de una roca piroclástica, es decir, se escogió ya al muestrear productos piroclásticos homogéneos, en los que su posible heterogeneidad natural (p.e., presencia de líticos accidentales incorporados en el proceso de erupción de la roca vol- cánica) fuera de incidencia mínima y en los que la naturaleza ya ha actuado (con el tipo de erupción volcánica desarrollada) mezclando y homogeneizando el producto natural. En todo caso, con un control textural y químico-mineralógico no más complejo que el usualmente empleado en el frente de corta de una mina a cielo abierto se puede garantizar la calidad y homogeneidad de la materia prima empleada.

\subsection{Ahorro en términos energéticos.}

Los vidrios volcánicos naturales son metaestables y tienden a sufrir transformaciones (cristalizaciones) en tiempos relativamente cortos en la escala geológica; es decir, es razonable pensar que rocas volcánicas de unos pocos centenares de miles de años (o con más motivo de algunos millones) pueden estar parcial o totalmente transformadas a silicatos, ya sea del grupo de los tectosilicatos (principalmente del grupo de las zeolitas, pero también feldespatos y fases cristalinas de la sílice) o de las arcillas. Esta capacidad de transformación es mayor cuanto mayor sea la superficie de interacción entre la roca y los fluidos acuosos existentes en el medio natural superficial, es decir, que rocas piroclásticas muy vesiculares y fragmentadas como las escogidas son francamente propensas a la transformación.

Los criterios de prospección iniciales ya evitaron las transformaciones generalizadas de los vidrios a arcillas, pero las zeolitizaciones son mucho más difícilmente reconocibles sobre el terreno, y su incidencia sobre el proceso de fabricación del cemento desconocida. Por este motivo se procedió a la caracterización de las rocas mediante DRX (acompañada lógicamente de estudio petrográfico mediante lámina delgada), con los resultados recogidos esquemáticamente en la tabla 2, y al rediseño de los experimentos de fabricación de los cementos en el laboratorio, concretamente, a la fabricación, además de sobre el producto vitrificado realizado en la fase inicial, sobre el producto crudo y también sobre un producto calcinado (roca volcánica llevada en horno a $800^{\circ} \mathrm{C}$ ), con los resultados técnicos antes expuestos (apartado 3.2, tabla 2). La elección de la calcinación a $800{ }^{\circ} \mathrm{C}$ parte simplemente de un paso previo ya desarrollado en el proceso de vitrificación (al objeto de eliminar los carbonatos naturales potencialmente presentes en las formulaciones del cemento) y debe entenderse más como un grado de economía en energía en el proceso que como la exploración sistemática y la consiguiente explotación máxima de las economías energéticas potenciales en el sistema, variando evidentemente de caso en caso según la roca natural empleada en el cemento (véase resultados de la resistencia a la compresión en la tabla 2).

Como el empleo de una calcinación como alternativa al proceso de vitrificación no conlleva las ventajas técnicas antes expresadas de ésta y es simplemente una elección de tipo económico en todo caso más costosa que el empleo del producto crudo, no es ocioso analizar las variaciones de comportamiento de los cementos desarrollados a partir de muestra cruda y calcinada e intentar determinar su origen.

Un estudio de los resultados recogidos en la tabla 2 nos permite extraer algunas conclusiones. Los resultados obtenidos sobre la muestra cruda muestran que existe un primer grupo de muestras que presentan resultados de resistencia a la compresión de alrededor de $50 \mathrm{MPa}$ (ES-03, LA-01, SA-01 y SA-07). Estas muestras en general presentan contenidos en elementos alcalinos iguales o inferiores al resto, hecho que demuestra que, al menos a partir de contenidos totales por 
encima del 8\% (expresado en forma de óxidos) el contenido en elementos alcalinos no es crítico para la obtención del cemento, contrariamente a lo pensado al inicio del proyecto a partir de la patente desarrollada sobre reactivos puros (2). Del mismo modo podemos decir que la relación $\mathrm{K} / \mathrm{Na}$ tampoco es crítica ya que es muy variable en todo el conjunto de muestras que han dado buenos resultados, y que en todo caso sólo pudiera ser un factor a controlar de cara a la durabilidad posterior del cemento. La relación $\mathrm{Si} / \mathrm{Al}$ sí que es marcadamente mayor en las muestras que mejores resultados proporcionan en crudo, pero la muestra LA-01 (con una atípica relación Si / Al originada por su anómalo contenido en $\mathrm{Al}$, véase tabla 1) demuestra por la vía de la excepción que este factor no es limitante, al menos en el rango de relaciones consideradas. La diferencia más notable entre estas muestras y el resto es su bajo contenido en volátiles, esencialmente agua, debido a que se trata de muestras casi totalmente desvitrificadas (o a un vidrio natural casi anhidro en el caso de la muestra ES-03) que han pasado a tener una mineralogía caracterizada por tectosilicatos relativamente estables (incluimos en este grupo la zeolita analcima). El resto de las muestras presentan contenidos elevados en agua que denotan que los vidrios originales de estas rocas han sido transformados, principalmente en minerales del grupo de las zeolitas, que presentan esta agua en su estructura cristalina y la liberan en su mayor parte en el intervalo $120-350{ }^{\circ} \mathrm{C}(19)$.

Las transformaciones inherentes a la calcinación del primer tipo de rocas no ofrecen diferencias en el caso del vidrio anhidro (ES-03), hay mejora en el caso de la muestra que contiene analcima (LA-O1) y hay empeoramiento notable en el resto, debido tanto a cambios de fase (ópalo C-T a cuarzo, adularia o microclina a feldespato de media-alta temperatura) como posiblemente a recristalización con incremento en el tamaño de grano.

En el resto de muestras se observa una evidente mejora con la calcinación, que conduce a la gradual desaparición de las estructuras de tipo zeolita (en el caso concreto de la phillipsita, a partir de $400^{\circ} \mathrm{C}$ ) y pérdida de agua estructural en la muestra, siendo una posible explicación para el peor resultado de la muestra LA-02 su más bajo contenido en álcalis (menor al $8 \%$ expresado en óxidos) y sílice que compensa la mayor presencia de Ca (véase tabla 1).

Estos controles químico-mineralógicos relativamente sencillos demuestran por lo tanto su efectividad tanto en la elaboración de criterios de selección de materias primas alcalinas económicas (y retroalimentan por lo tanto los criterios de prospección desarrollados en las fases iniciales del proyecto) como en el diseño del tipo de proceso de fabricación del cemento y consecuentemente en la economía de éste.

Aunque todo el conjunto de diez rocas volcánicas estudiadas son susceptibles de ser empleadas como materia prima en los cementos, podemos resumir que rocas alcalinas con contenidos alrededor del $8 \%$ (o superiores) en sumatorio de óxidos alcalinos, y caracterizadas por ser relativamente anhidras, estar desvitrificadas y poseer una mineralogía caracterizada por fases cristalinas de la sílice y feldespatos, generados a temperaturas inferiores a las magmáticas (es decir, en procesos naturales de desvitrificación) son a priori más interesantes (en términos económicos, no requieren calcinación) que rocas equivalentes total o parcialmente zeolitizadas. Parece evidente que esta mayor capacidad reactiva para la formulación del cemento tiene su origen en la disposición estructural de los elementos potencialmente reactivos en la roca volcánica y que es susceptible de ser estudiada en un futuro por MAS-MNR $\left({ }^{29} \mathrm{Si},{ }^{27} \mathrm{Al}\right)$.

\section{AGRADECIMIENTOS}

Este estudio ha sido financiado por la European Commission (contrato BRE2-CT93-0559). Además ha disfrutado de cofinanciación por parte española (proyecto CICYT AMB94-1529). Los autores desean expresar su agradecimiento al personal científico y técnico que en las diferentes instituciones participantes ha colaborado en diferentes partes del proyecto, así como a los responsables de los laboratorios subcontratados. Igualmente, por lo que se refiere específicamente a las campañas de muestreo agradecen particularmente la ayuda aportada por G. De Casa (La Sapienza, Roma), A. Filippidis (Aristotle Univ., Thessaloniki, Grecia), J.Mangas y F.J.Pérez Torrado (ULPGC, España). Los interesados en información detallada sobre la base de datos geológicos pueden contactar directamente con el primer autor de la nota, mientras que los que deseen más informaciones sobre las pruebas tecnológicas de los cementos pueden contactar directamente a otro de los coautores (J.D.). El Geological Survey of Japan suministró un juego completo de patrones certificados de rocas naturales que fueron empleadas como control interno de los análisis de FRX.

\section{BIBLIOGRAFIA}

1. J.Davidovits, D.Gimeno, C.Marini, A.Rinaldi, Ph.Rocher, S.Tocco. "BRITE EURAM BE-7355-93, Contract N ${ }^{\circ}$ BRE2-CT93-0559, GEOCISTEM, cost effective GEOpolymeric Cements for Innocuos Stabilization of Toxic EleMents. Final Technical Report from January 1, 1994 to February 28, 1997", 72 pp, Informe inédito para la Comisión Europea, 1997.

2. Patente Europea 0338060 .

3. J.Davidovits, L.Buzzi, Ph.Rocher, D.Gimeno, C.Marini, S.Tocco. "Geopolymeric cement basedt onlow cost geologic materials. Results from the european research project GEOCISTEM". 83-96, in: 2nd International Conference June 30-July 2, Geopolymère'99 Proceedings, Saint Quentin, Francia, ISBN 2-902933-14-2, 1999.

4. Patente francesa FR 97-00332.

5. M.Frantini, L.Leoni, M.Saitta. "Revisione di una metodologia analitica per fluorescenza-X basata sulla correzione completa degli effetti di matrice". Rend.S.I.M.P. XXXI (2):365-378, (1975).

6. R.W.Le Maître (Ed). "A classification of igneous rocks and glossary of ter$\mathrm{ms}$. Recommendations of the International Union of Geological Sciences, Subcommission on the systematics of igneous rocks". 193 pp. Blackwell Scientific Publications, Oxford, 1989.

7. D.Clarke. Dept. Earth Sciences, Univ. of Newfoundland, Canada, 1987-1990.

8. D.Gimeno, M.Pugès. "Caracterización química de la vidriera de Sant Pere i Sant Jaume (segundo cuarto del s. XIV, Monestir de Pedralbes, Barcelona)". Bol.Soc.Esp.Cerám.Vidrio 41 (2):225-231, (2002).

9. A.Assorgia, A.Fadda, D.Gimeno, V.Morra, L.Otelli, F.A.Secchi. "Le succession ignimbritiche terziarie del Sulcis (Sardegna Sud-Occidentale)". Bol.Soc.Geol.It. 45: 951-963, (1990).

10. G.Engelhardt, D.Michel "High-Resolution solid-state NMR of silicates and zeolites". John Wiley \& Sons, Chichester, 485 pp, 1987.

11. J.Davidovits "Geopolymers. Inorganic polymeric new materials" Jour. Thermal Analysis 37:1633-1659, (1991).

12. R.Aiello, C.Colella. "La sintessi delle zeoliti". Rend.S.I.M.P XXXI (2): 565-584, (1975).

13. R.Aielo, E.Franco. "Formazione di zeoliti per trasformazione di halloysite e montmorillonite a bassa temperatura ed in ambiente alcalino". Rend.Acad.Sci.Fis. e Mat. Della Soc.Naz. di Sci.Lettere ed Arti di Napoli 35 (4): 165-192, (1968).

14. R.A.Clifton "Natural and synthetic zeolites". U.S.Bureau of Mines Information Circular 9140: 21 pp, 1987.

15. Aznar,A,J, and La Iglesia,A. “Obtención de zeolitas a partir de arcillas aluminosas españolas". Bol.Geol Minero 96: 541-549, (1985).

16. J.Sanz, A.Madani, J.M.Serratosa, J.S.Moya, S.Aza "Aluminium -27 and Silicon-20 Magic-Angle Spinning Nuclear Magnetic Resonance study of the kaolinite-mullite transformation". J.Am.Ceram.Soc. 71, (10): C418-421, (1988).

17. D.Akokelar, A.Chaffee, R.F.Howe "The transformation of kaolin to low-silica $X$ zeolite". Zeolites 19: 359-365, (1997).

18. E.Lippmaa, A.Samoson, M.Magi "High resolution ${ }^{29} \mathrm{Al}$ MNR of aluminosilicates" J.Am.Chem.Soc. 108, 1730-1735 (1986).

19. G.Gottardi, E.Galli. “Natural zeolites”.409 pp. Springer Verlag, Berlin, 1985.

Recibido: 11.11 .00

Aceptado: 24.02 .03 